\title{
Stochastic Comparison of Residual Life of Order Statistics at Random Time
}

\author{
Fengxiao Wang \\ College of Mathematics and Statistics, Kashigar University, Kashi, 844000, China
}

\begin{abstract}
Stochastic comparison on order statistics has been paid lots of attention recently. This paper devotes to investigating the stochastic comparison properties of residual life and inactivity time at random time. Preserved properties of some stochastic comparisons on the residual life and inactivity time at random time of order statistics are established. When the number of random variables is random, the stochastic comparison results are presented as well.
\end{abstract}

Keywords-order statistics; RLRT; ITRT; likelihood ratio order; hazard rate order

\section{INTRODUCTION}

Order statistics play a central role in statistics and a lot of work has been done in the literature on different aspects of this problem. Let $X_{1: \mathrm{n}} \leqslant X_{2: \mathrm{n}} \leqslant \cdots \leqslant X_{\text {n:n }}$ be the order statistics corresponding $X_{1}, X_{2}, \cdots, X_{\mathrm{n}}$, a sample of size $n$ form the population $X$. In auction theory, $X_{\mathrm{i}: \mathrm{n}}$ can be regarded as the $i$-th bidding price if the valuations of bidders are independent and identical (i.i.d.) random variables. In reliability, the $i$-th order statistics $X_{\text {i:n }}$ gives the total life of a $(n-i+1)$-out-of- $n$ system with i.i.d. components of life $X$. In the past decades, much attention has been paid to investigate the properties of order statistics. For more details of these properties, readers can refer to Davia and Nagaraja(2003) for a comprehensive discussion.

Before proceeding to state our main results, we firstly recall several criteria for comparison random variables and some notions which will be used in this paper. These criteria widely used in describing notions of aging in reliability theory. Let $X$ and $Y$ be two random variables with absolutely continuous cumulative distribution functions $F(x)$ and $G(x)$, and their density functions are $f(x), g(x)$, respectively.

Definition 1.1 (a) $Y$ is said to be larger than $X$ in the hazard rate order (denoted by $X \leq_{h r} Y$ ) if $\bar{F}(x) / \bar{G}(x)$ decreases in $x$.

(b) $Y$ is said to be larger than $X$ in the reversed hazard rate order (denoted by $X \leq_{r h} Y$ ) if $F(x) / G(x)$ decreases in $x$.

(c) $Y$ is said to be larger than $X$ in the likelihood ratio order (denoted by $X \leq_{l r} Y$ ) if $f(x) / g(x)$ decreases over the union of the two supports.

For convenience, relations among these orderings are presented in the following:

$$
X \leq_{l r} Y \Rightarrow X \leq_{h r(r h)} Y \Rightarrow X \leq_{s t} Y
$$

Assume $X$ and $Y$, two component lifetimes, to be random variables. The residual life and the inactivity time of the used components with age $t>0$ are respectively defined as $X_{t}=(X$ $t \mid X>t), X_{(t)}=(t-X \mid X<t)$. Series systems and parallel systems are two familiar reliability structures. In practical situation, one often meets used system composed of new units. Li and Zhang (2002)[1] have proved that the life of a parallel or series system composed of used i.i.d. elements is stochastically larger than that of a used parallel or series system. Pellerey and Petakos (2002)[2] obtained a more general conclusion, which asserts that the life of a coherent system composed of used elements is stochastically larger than that of a used coherent system. Li and $\mathrm{Lu}$ (2003)[3] established some stochastic comparison results on their inactivity time and residual life for parallel or series system, respectively.

Now we briefly review the concepts of the residual life and inactivity time of $X$ at a random time. Let $T$ be a random variable with distribution function $H(t)$, and probability density function $h(t)$. Further, suppose that $X$ and $T$ are statistically independent. The random variable $X_{T}=(X-T \mid X>T)$ is called the residual life of $X$ at random time $T$ (RLRT). The random variable $X_{(T)}=(T-X \mid X<T)$ is called the inactivity time of $X$ at random time $T$ (ITRT). The idle time of the server in a GI/G/1 queuing system can be expressed as a RLRT. In reliability theory, if $X$ is regarded as the total random life of a warm stand-by unit with an age of $T$. For more details about residual life and inactivity time and RLRT, see [4-15].

The concept of residual life and inactivity time at some random time plays an important role in reliability, survival analysis and life testing. In this paper, we present some results for stochastic comparisons of residual life and inactivity time of order statistics at random time. In particular, we establish some stochastic comparisons the residual life and inactivity time of order statistics at random time. Some comparisons of the inactivity time and the residual life at random time are presented as well.

\section{MAIN RESULTS}

Let $F_{j: n}(x)$ be the distribution of the $j$-th order statistic in a sample of $n$ from $F$. Then $F_{j: n}(x)=B_{j: n}[F(x)]$ for $x>0$, where

$$
B_{j: n}(p)=\frac{n !}{(j-1) !(n-j) !} \int_{0}^{p} u^{j-1}(1-u)^{n-j} \mathrm{~d} u
$$

for $0<p<1$. In actuarial science, $X_{j: n}$ can be regarded as $j$-th bid price if the valuations of bidders are i.i.d. random variable $X$. In 
reliability, $j$-th order statistics $X_{j: n}$ can be regarded as the life of a $(n-j+1)$-out-of- $n$ systems with i.i.d. components of life $X$.

Before stating our main conclusions, we firstly introduce the following lemmas (See [4]) which will be used in sequel.

Lemma 2.1 Let $X$ and $Y$ be two independent random variables. Let $\phi_{1}$ and $\phi_{2}$ be two bivariate functions. Denote

$$
\Delta \phi_{21}(x, y)=\phi_{2}(x, y)-\phi_{1}(x, y) \text {. }
$$

Then $X \leqslant_{l r} Y$ if, and only if, $E \phi_{1}(X, Y) \leq E \phi_{2}(X, Y)$ for all $\phi_{1}$ and $\phi_{2}$ such that $\Delta \phi_{21}(x, y) \geq 0$ whenever $x \leq y$, and $\Delta \phi_{21}(x, y) \geq-\Delta \phi_{21}(y, x)$ whenever $x \leq y$.

Lemma 2.2 Let $X$ and $Y$ be two independent random variables. Let $\phi_{1}$ and $\phi_{2}$ be two bivariate functions. Denote

$$
\Delta \phi_{21}(x, y)=\phi_{2}(x, y)-\phi_{1}(x, y) .
$$

Then $X \leqslant_{h r} Y$ if, and only if, $E \phi_{1}(X, Y) \leq E \phi_{2}(X, Y)$ for all $\phi_{1}$ and $\phi_{2}$ such that ,for each $x, \Delta \phi_{21}(x, y)$ increases in $y$ on $\{y \geq x\}$, and such that $\Delta \phi_{21}(x, y) \geq-\Delta \phi_{21}(y, x)$ whenever $x \leq y$.

Lemma 2.3 Let $X$ and $Y$ be two independent random variables. Let $\phi_{1}$ and $\phi_{2}$ be two bivariate functions. Denote

$$
\Delta \phi_{21}(x, y)=\phi_{2}(x, y)-\phi_{1}(x, y)
$$

Then $X \leqslant_{\mathrm{hr}} Y$ if, and only if, $E \phi_{1}(X, Y) \leq E \phi_{2}(X, Y)$ for all $\phi_{1}$ and $\phi_{2}$ such that ,for each $y, \Delta \phi_{21}(x, y)$ decreases in $x$ on $\{y \geq x\}$, and such that $\Delta \phi_{21}(x, y) \geq-\Delta \phi_{21}(y, x)$ whenever $x \leq y$.

Now, we present our main results.

Theorem 2.1 Assume that $X$ and $Y$ are independent (not necessarily identical), $T$ is a non-negative random variable, and $T, X$ and $Y$ are independent. If $X \leqslant l r Y$, then for any $1 \leqslant i \leqslant n$,

$$
\left(X_{i: n}\right)_{t} \leq_{l r}\left(Y_{i: n}\right)_{t}
$$

Theorem 2.2 Assume that $X$ and $Y$ are independent (not necessarily identical), $T$ is a non-negative random variable, and $T, X$ and $Y$ are independent. If $X \leqslant_{l r} Y$, then for any $1 \leqslant i \leqslant n$,

$$
\left(X_{i: n}\right)_{T} \leq_{l r}\left(Y_{i: n}\right)_{T}
$$

Proof Since $X \leqslant_{l r} Y$, then

$$
\left(X_{i: n}\right)_{t} \leq_{l r}\left(Y_{i: n}\right)_{t}
$$

Suppose $\phi_{1}(x, y)$ and $\phi_{2}(x, y)$ satisfy Lemma 1 , by (1) and Lemma 1 we have

$$
E \phi_{1}\left(\left(X_{i: n}\right)_{t},\left(Y_{i: n}\right)_{t}\right) \leq E \phi_{2}\left(\left(X_{i: n}\right)_{t},\left(Y_{i: n}\right)_{t}\right)
$$

Hence

$$
\begin{aligned}
E \phi_{1} & \left(\left(X_{i: n}\right)_{T},\left(Y_{i: n}\right)_{T}\right)=E\left[E\left(\phi_{1}\left(\left(X_{i: n}\right)_{T},\left(Y_{i: n}\right)_{T}\right) \mid T\right)\right] \\
& =\int_{0}^{+\infty} E\left(\phi_{1}\left(\left(X_{i: n}\right)_{T},\left(Y_{i: n}\right)_{T}\right) \mid T=t\right) h(t) d t \\
& =\int_{0}^{+\infty} E\left(\phi_{1}\left(\left(X_{i: n}\right)_{t},\left(Y_{i: n}\right)_{t}\right)\right) h(t) d t \\
& \leq \int_{0}^{+\infty} E\left(\phi_{2}\left(\left(X_{i: n}\right)_{t},\left(Y_{i: n}\right)_{t}\right)\right) h(t) d t \\
& =\int_{0}^{+\infty} E\left(\phi_{2}\left(\left(X_{i: n}\right)_{T},\left(Y_{i: n}\right)_{T}\right) \mid T=t\right) h(t) d t \\
& =E\left[E\left(\phi_{1}\left(\left(X_{i: n}\right)_{T},\left(Y_{i: n}\right)_{T}\right) \mid T\right)\right] \\
& =E \phi_{2}\left(\left(X_{i: n}\right)_{T},\left(Y_{i: n}\right)_{T}\right)
\end{aligned}
$$

By Lemma 1, $\left(X_{i: n}\right)_{T} \leq_{l r}\left(Y_{i: n}\right)_{T}$.

Corollary 2.1 Let $X_{1}, X_{2}, \cdots, X_{n}$ and $Y_{1}, Y_{2}, \cdots, Y_{n}$ are i.i.d. copies of $X$ and $Y$, respectively, and $T$ is a non-negative random variable. If $X \leqslant{ }_{l r} Y$, then

$$
\begin{aligned}
& \left(\max \left\{X_{1}, X_{2}, \cdots, X_{n}\right\}\right)_{T} \leq_{l r}\left(\max \left\{Y_{1}, Y_{2}, \cdots, Y_{n}\right\}\right)_{T} \\
& \left(\min \left\{X_{1}, X_{2}, \cdots, X_{n}\right\}\right)_{T} \leq_{l r}\left(\min \left\{Y_{1}, Y_{2}, \cdots, Y_{n}\right\}\right)_{T}
\end{aligned}
$$

Theorem 2.3 Assume that $X$ and $Y$ are independent (not necessarily identical), $\mathrm{N}$ be a positive integer-valued random variable which is independent of the $X$ and $Y$ are independent.

$$
\text { If } X \leqslant_{l r} Y \text {, then }\left(X_{i: N}\right)_{t} \leq_{l r}\left(Y_{i: N}\right)_{t}
$$

Proof Since $X \leqslant_{l r} Y$, then $\left(X_{i: n}\right)_{t} \leq_{l r}\left(Y_{i: n}\right)_{t}$. Suppose $\varphi_{1}(x, y)$ and $\varphi_{2}(x, y)$ satisfy Lemma 1 , then

$$
E \varphi_{1}\left(\left(X_{i: n}\right)_{t},\left(Y_{i: n}\right)_{t}\right) \leq E \varphi_{2}\left(\left(X_{i: n}\right)_{t},\left(Y_{i: n}\right)_{t}\right)
$$

Hence

$$
\begin{aligned}
E & \varphi_{1}\left(\left(X_{i: N}\right)_{t},\left(Y_{i: N}\right)_{t}\right)=E\left[E\left(\phi_{1}\left(\left(X_{i: N}\right)_{t},\left(Y_{i: N}\right)_{t}\right) \mid N\right)\right] \\
= & \sum_{n=1}^{+\infty} E\left(\varphi_{1}\left(\left(X_{i: N}\right)_{t},\left(Y_{i: N}\right)_{t}\right) \mid N=n\right) P(N=n) \\
= & \sum_{n=1}^{+\infty} E\left(\varphi_{1}\left(\left(X_{i: n}\right)_{t},\left(Y_{i: n}\right)_{t}\right)\right) P(N=n) \\
\leq & \sum_{n=1}^{+\infty} E\left(\varphi_{2}\left(\left(X_{i: n}\right)_{t},\left(Y_{i: n}\right)_{t}\right)\right) P(N=n) \\
= & \sum_{n=1}^{+\infty} E\left(\varphi_{2}\left(\left(X_{i: N}\right)_{t},\left(Y_{i: N}\right)_{t}\right) \mid N=n\right) P(N=n) \\
= & E\left[E\left(\phi_{1}\left(\left(X_{i: N}\right)_{t},\left(Y_{i: N}\right)_{t}\right) \mid N\right)\right] \\
= & E \varphi_{2}\left(\left(X_{i: N}\right)_{t},\left(Y_{i: N}\right)_{t}\right)
\end{aligned}
$$

By Lemma 1, $\left(X_{i: N}\right)_{t} \leq_{l r}\left(Y_{i: N}\right)_{t}$. 
Theorem 2.4 Assume that $X$ and $Y$ are independent (not necessarily identical), $T$ is a non-negative random variable, $\mathrm{N}$ be a positive integer-valued random variable which is independent of the $X$ and $Y$, and $T, X$ and $Y$ are independent. If $X \leqslant_{l r} Y$, then

$$
\left(X_{i: N}\right)_{T} \leq_{l r}\left(Y_{i: N}\right)_{T}
$$

Proof Since $X \leqslant_{l r} Y$, by theorem $2.3\left(X_{i: N}\right)_{t} \leq_{l r}\left(Y_{i: N}\right)_{t}$.

Suppose $\phi_{1}(x, y)$ and $\phi_{2}(x, y)$ satisfy Lemma 1 , by Lemma 1 we have

Hence

$$
E \phi_{1}\left(\left(X_{i: N}\right)_{t},\left(Y_{i: N}\right)_{t}\right) \leq E \phi_{2}\left(\left(X_{i: N}\right)_{t},\left(Y_{i: N}\right)_{t}\right)
$$

$$
\begin{aligned}
& E \phi_{1}\left(\left(X_{i: N}\right)_{T},\left(Y_{i: N}\right)_{T}\right)=E\left[E\left(\phi_{1}\left(\left(X_{i: N}\right)_{T},\left(Y_{i: N}\right)_{T}\right) \mid T\right)\right] \\
& \quad=\int_{0}^{+\infty} E\left(\phi_{1}\left(\left(X_{i: N}\right)_{T},\left(Y_{i: N}\right)_{T}\right) \mid T=t\right) h(t) d t \\
& =\int_{0}^{+\infty} E\left(\phi_{1}\left(\left(X_{i: N}\right)_{t},\left(Y_{i: N}\right)_{t}\right)\right) h(t) d t \\
& \quad \leq \int_{0}^{+\infty} E\left(\phi_{2}\left(\left(X_{i: N}\right)_{t},\left(Y_{i: N}\right)_{t}\right)\right) h(t) d t \\
& =\int_{0}^{+\infty} E\left(\phi_{2}\left(\left(X_{i: N}\right)_{T},\left(Y_{i: N}\right)_{T}\right) \mid T=t\right) h(t) d t \\
& =E\left[E\left(\phi_{1}\left(\left(X_{i: N}\right)_{T},\left(Y_{i: N}\right)_{T}\right) \mid T\right)\right] \\
& =E \phi_{2}\left(\left(X_{i: N}\right)_{T},\left(Y_{i: N}\right)_{T}\right)
\end{aligned}
$$

By Lemma $1,\left(X_{i: N}\right)_{T} \leq_{l r}\left(Y_{i: N}\right)_{T}$.

Corollary 2.2 Let $X_{1}, X_{2}, \cdots$, and $Y_{1}, Y_{2}, \cdots$, are i.i.d. copies of $X$ and $Y$, respectively. Let $\mathrm{N}$ be a positive integervalued random variable which is independent of the $X$ and $Y$. If $X \leqslant_{l r} Y$, then

$$
\begin{aligned}
& \left(\max \left\{X_{1}, X_{2}, \cdots, X_{N}\right\}\right)_{T} \leq_{l r}\left(\max \left\{Y_{1}, Y_{2}, \cdots, Y_{N}\right\}\right)_{T} \\
& \left(\min \left\{X_{1}, X_{2}, \cdots, X_{N}\right\}\right)_{T} \leq_{l r}\left(\min \left\{Y_{1}, Y_{2}, \cdots, Y_{N}\right\}\right)_{T}
\end{aligned}
$$

Theorem 2.5 Assume that $X$ and $Y$ are independent (not necessarily identical), $T$ is a non-negative random variable, and $T, X$ and $Y$ are independent. If $X \leqslant_{h r} Y$, then

$$
\left(\min \left\{X_{1}, X_{2}, \cdots, X_{n}\right\}\right)_{T} \leq_{h r}\left(\min \left\{Y_{1}, Y_{2}, \cdots, Y_{n}\right\}\right)_{T} .
$$

Proof For fixed $\mathrm{t}>0$, Let $M_{t}=\left(\min \left\{X_{1}, X_{2}, \cdots, X_{n}\right\}\right)_{t}$, $N_{t}=\left(\min \left\{Y_{1}, Y_{2}, \cdots, Y_{n}\right\}\right)_{t}$, then

$$
\begin{aligned}
& P\left(M_{t}>x\right)=\frac{\bar{F}_{1: n}(x+t)}{\bar{F}_{1: n}(t)}=\frac{\bar{F}^{n}(x+t)}{\bar{F}^{n}(t)}, \\
& P\left(N_{t}>x\right)=\frac{\bar{G}_{1: n}(x+t)}{\bar{G}_{1: n}(t)}=\frac{\bar{G}^{n}(x+t)}{\bar{G}^{n}(t)}
\end{aligned}
$$

Since $X \leqslant_{h r} Y$, then $\bar{G}(x) / \bar{F}(x)$ is increasing in $x$. Thus $P\left(N_{1}>x\right) / P\left(M_{1}>x\right)$ is increasing in $x$, hence

$$
\left(\min \left\{X_{1}, X_{2}, \cdots, X_{n}\right\}\right)_{t} \leq_{h r}\left(\min \left\{Y_{1}, Y_{2}, \cdots, Y_{n}\right\}\right)_{t}
$$

Suppose $\varphi_{1}(x, y)$ and $\varphi_{2}(x, y)$ satisfy Lemma 2.2 , then

$$
\begin{array}{r}
E \varphi_{1}\left(M_{t}, N_{t}\right) \leq E \varphi_{2}\left(M_{t}, N_{t}\right) \\
\text { Let } \quad M_{T}=\left(\min \left\{X_{1}, \cdots, X_{n}\right\}\right)_{T} \\
N_{T}=\left(\min \left\{Y_{1}, \cdots, Y_{n}\right\}\right)_{T}
\end{array}
$$

Hence

$$
\begin{aligned}
E & \left.\varphi_{1}\left(M_{T}, N_{T}\right)=E\left[E \varphi_{1}\left(M_{T}, N_{T}\right) \mid T\right)\right] \\
& \left.=\int_{0}^{+\infty} E\left(\varphi_{1}\left(M_{T}, N_{T}\right)\right) \mid T=t\right) h(t) d t \\
& =\int_{0}^{+\infty} E\left(\varphi_{1}\left(M_{t}, N_{t}\right)\right) h(t) d t \\
& \leq \int_{0}^{+\infty} E\left(\varphi_{2}\left(M_{t}, N_{t}\right)\right) h(t) d t \\
& \left.=\int_{0}^{+\infty} E\left(\varphi_{2}\left(M_{T}, N_{T}\right)\right) \mid T=t\right) h(t) d t \\
& \left.=E\left[E\left(\varphi_{2}\left(M_{T}, N_{T}\right)\right) \mid T\right)\right]=E \varphi_{2}\left(M_{T}, N_{T}\right)
\end{aligned}
$$

By Lemma 2.2, $M_{T} \leq_{h r} N_{T}$, that is

$$
\left(\min \left\{X_{1}, X_{2}, \cdots, X_{n}\right\}\right)_{T} \leq_{h r}\left(\min \left\{Y_{1}, Y_{2}, \cdots, Y_{n}\right\}\right)_{T}
$$

Corollary 2.3 Let $X_{1}, X_{2}, \cdots$, and $Y_{1}, Y_{2}, \cdots$, are i.i.d. copies of $X$ and $Y$, respectively. If $X \leqslant_{h r} Y$, then

$$
\left(\min \left\{X_{1}, X_{2}, \cdots, X_{n}\right\}\right)_{t} \leq_{h r}\left(\min \left\{Y_{1}, Y_{2}, \cdots, Y_{n}\right\}\right)_{t}
$$

Theorem 2.6 Assume that $X$ and $Y$ are independent (not necessarily identical), $T$ is a non-negative random variable, $\mathrm{N}$ be a positive integer-valued random variable which is independent of the $X$ and $Y$, and $T, X$ and $Y$ are independent. If $X \leqslant_{h r} Y$, then

$$
\left(\min \left\{X_{1}, X_{2}, \cdots, X_{N}\right\}\right)_{t} \leq_{h r}\left(\min \left\{Y_{1}, Y_{2}, \cdots, Y_{N}\right\}\right)_{t}
$$

Theorem 2.7 Assume that $X$ and $Y$ are independent (not necessarily identical), $T$ is a non-negative random variable, and $T, X$ and $Y$ are independent. If $X \leqslant{ }_{l r} Y$, then for any $1 \leqslant i \leqslant n$,

$$
\left(X_{i: n}\right)_{(T)} \geq_{l r}\left(Y_{i: n}\right)_{(T)}
$$

Corollary 2.4 Let $X_{1}, X_{2}, \cdots, X_{n}$ and $Y_{1}, Y_{2}, \cdots, Y_{n}$ are i.i.d. copies of $X$ and $Y$, respectively, and $T$ is a non-negative random variable. If $X \leqslant_{l r} Y$, then

$$
\begin{aligned}
& \left(\max \left\{X_{1}, X_{2}, \cdots, X_{n}\right\}\right)_{(T)} \geq_{l r}\left(\max \left\{Y_{1}, Y_{2}, \cdots, Y_{n}\right\}\right)_{(T)} \\
& \left(\min \left\{X_{1}, X_{2}, \cdots, X_{n}\right\}\right)_{(T)} \geq_{l r}\left(\min \left\{Y_{1}, Y_{2}, \cdots, Y_{n}\right\}\right)_{(T)}
\end{aligned}
$$

Theorem 2.8 Assume that $X$ and $Y$ are independent (not necessarily identical), $T$ is a non-negative random variable, $\mathrm{N}$ be a positive integer-valued random variable which is independent of the $X$ and $Y$, and $T, X$ and $Y$ are independent. If $X \leqslant_{l r} Y$, then

$$
\left(X_{i: N}\right)_{(T)} \geq_{l r}\left(Y_{i: N}\right)_{(T)}
$$

Proof Since $X \leqslant_{l r} Y$, then $\left(X_{i: n}\right)_{(t)} \geq_{l r}\left(Y_{i: n}\right)_{(t)}$. 
Suppose $\varphi_{1}(x, y)$ and $\varphi_{2}(x, y)$ satisfy Lemma 2.1 , then

$$
E \varphi_{1}\left(\left(X_{i: n}\right)_{(t)},\left(Y_{i: n}\right)_{(t)}\right) \geq E \varphi_{2}\left(\left(X_{i: n}\right)_{(t)},\left(Y_{i: n}\right)_{(t)}\right)
$$

Hence

$$
\begin{aligned}
& E \varphi_{1}\left(\left(X_{i: N}\right)_{t},\left(Y_{i: N}\right)_{(t)}\right)=E\left[E\left(\varphi_{1}\left(\left(X_{i: N}\right)_{(t)},\left(Y_{i: N}\right)_{(t)}\right) \mid N\right)\right] \\
& =\sum_{n=1}^{+\infty} E\left(\varphi_{1}\left(\left(X_{i: N}\right)_{(t)},\left(Y_{i: N}\right)_{(t)}\right) \mid N=n\right) P(N=n) \\
& =\sum_{n=1}^{+\infty} E\left(\varphi_{1}\left(\left(X_{i: n}\right)_{(t)},\left(Y_{i: n}\right)_{(t)}\right)\right) P(N=n) \\
& \geq \sum_{n=1}^{+\infty} E\left(\varphi_{2}\left(\left(X_{i: n}\right)_{(t)},\left(Y_{i: n}\right)_{(t)}\right)\right) P(N=n) \\
& =\sum_{n=1}^{+\infty} E\left(\varphi_{2}\left(\left(X_{i: N}\right)_{(t)},\left(Y_{i: N}\right)_{(t)}\right) \mid N=n\right) P(N=n) \\
& =E\left[E\left(\phi_{1}\left(\left(X_{i: N}\right)_{(t)},\left(Y_{i: N}\right)_{(t)}\right) \mid N\right)\right] \\
& =E \varphi_{2}\left(\left(X_{i: N}\right)_{(t)},\left(Y_{i: N}\right)_{(t)}\right)
\end{aligned}
$$

By Lemma 2. 1, $\left(X_{i: N}\right)_{(t)} \geq_{l r}\left(Y_{i: N}\right)_{(t)}$.

Suppose $\phi_{1}(x, y)$ and $\phi_{2}(x, y)$ satisfy Lemma 1 , by (3) and Lemma 2.1 we have

$$
E \phi_{1}\left(\left(X_{i: N}\right)_{(t)},\left(Y_{i: N}\right)_{(t)}\right) \leq E \phi_{2}\left(\left(X_{i: N}\right)_{(t)},\left(Y_{i: N}\right)_{(t)}\right)
$$

Hence

$$
\begin{aligned}
E \phi_{1} & \left(\left(X_{i: N}\right)_{(T)},\left(Y_{i: N}\right)_{(T)}\right)=E\left[E\left(\phi_{1}\left(\left(X_{i: N}\right)_{(T)},\left(Y_{i: N}\right)_{(T)}\right) \mid T\right)\right] \\
& =\int_{0}^{+\infty} E\left(\phi_{1}\left(\left(X_{i: N}\right)_{(T)},\left(Y_{i: N}\right)_{(T)}\right) \mid T=t\right) h(t) d t \\
& =\int_{0}^{+\infty} E\left(\phi_{1}\left(\left(X_{i: N}\right)_{(t)},\left(Y_{i: N}\right)_{(t)}\right)\right) h(t) d t \\
& \geq \int_{0}^{+\infty} E\left(\phi_{2}\left(\left(X_{i: N}\right)_{(t)},\left(Y_{i: N}\right)_{(t)}\right)\right) h(t) d t \\
& =\int_{0}^{+\infty} E\left(\phi_{2}\left(\left(X_{i: N}\right)_{(T)},\left(Y_{i: N}\right)_{(T)}\right) \mid T=t\right) h(t) d t \\
& =E\left[E\left(\phi_{1}\left(\left(X_{i: N}\right)_{(T)},\left(Y_{i: N}\right)_{(T)}\right) \mid T\right)\right] \\
& =E \phi_{2}\left(\left(X_{i: N}\right)_{(T)},\left(Y_{i: N}\right)_{(T)}\right) \\
& \text { By Lemma } 1,\left(X_{i: N}\right)_{(T)} \geq_{l r}\left(Y_{i: N}\right)_{(T)} .
\end{aligned}
$$

Corollary 2.5 Let $X_{1}, X_{2}, \cdots$, and $Y_{1}, Y_{2}, \cdots$, are i.i.d. copies of $X$ and $Y$, respectively. Let $\mathrm{N}$ be a positive integervalued random variable which is independent of the $X$ and $Y$. If $X \leqslant_{l r} Y$, then

$$
\begin{aligned}
& \left(\max \left\{X_{1}, X_{2}, \cdots, X_{N}\right\}\right)_{(T)} \leq_{l r}\left(\max \left\{Y_{1}, Y_{2}, \cdots, Y_{N}\right\}\right)_{(T)} \\
& \left(\min \left\{X_{1}, X_{2}, \cdots, X_{N}\right\}\right)_{(T)} \leq_{l r}\left(\min \left\{Y_{1}, Y_{2}, \cdots, Y_{N}\right\}\right)_{(T)}
\end{aligned}
$$

Theorem 2.9 Let $X_{1}, X_{2}, \cdots$, and $Y_{1}, Y_{2}, \cdots$, are i.i.d. copies of $X$ and $Y$, respectively. If $X \leqslant_{r h} Y$, then

$$
\left(\max \left\{X_{1}, X_{2}, \cdots, X_{n}\right\}\right)_{(t)} \leq_{h r}\left(\max \left\{Y_{1}, Y_{2}, \cdots, Y_{n}\right\}\right)_{(t)}
$$

Theorem 2.10 Assume that $X$ and $Y$ are independent (not necessarily identical). If $X \leqslant_{r h} Y$, then

$$
\left(\max \left\{X_{1}, X_{2}, \cdots, X_{N}\right\}\right)_{(t)} \geq_{h r}\left(\max \left\{Y_{1}, Y_{2}, \cdots, Y_{N}\right\}\right)_{(t)}
$$

Theorem 2.11 Assume that $X$ and $Y$ are independent (not necessarily identical), $T$ is a non-negative random variable, and $T, X$ and $Y$ are independent. If $X \leqslant_{r h} Y$, then

$$
\begin{aligned}
& \left(\max \left\{X_{1}, X_{2}, \cdots, X_{n}\right\}\right)_{(T)} \geq_{h r}\left(\max \left\{Y_{1}, Y_{2}, \cdots, Y_{n}\right\}\right)_{(T)} \\
& \text { Proof Let } A_{T}=\left(\max \left\{X_{1}, X_{2}, \cdots, X_{n}\right\}\right)_{(T)}, \\
& B_{T}=\left(\max \left\{Y_{1}, Y_{2}, \cdots, Y_{n}\right\}\right)_{(T)}, \\
& A_{t}=\left(\max \left\{X_{1}, X_{2}, \cdots, X_{n}\right\}\right)_{(t)}, \\
& B_{t}=\left(\max \left\{Y_{1}, Y_{2}, \cdots, Y_{n}\right\}\right)_{(t)},
\end{aligned}
$$

Since $X \leqslant_{r h} Y$, by theorem2.10,we have $A_{t} \geq_{h r} B_{t}$

Suppose $\varphi_{1}(x, y)$ and $\varphi_{2}(x, y)$ satisfy Lemma 2.2, then

$$
E \varphi_{1}\left(A_{t}, B_{t}\right) \geq E \varphi_{2}\left(A_{t}, B_{t}\right)
$$

Hence

$$
\begin{aligned}
E \varphi_{1} & \left(A_{T}, B_{T}\right)=E\left[E\left(\varphi_{1}\left(A_{T}, B_{T}\right) \mid T\right)\right] \\
& \left.=\int_{0}^{+\infty} E\left(\varphi_{1}\left(A_{T}, B_{T}\right)\right) \mid T=t\right) h(t) \mathrm{d} t \\
& =\int_{0}^{+\infty} E\left(\varphi_{1}\left(A_{t}, B_{t}\right)\right) h(t) \mathrm{d} t \\
& \geq \int_{0}^{+\infty} E\left(\varphi_{2}\left(A_{t}, B_{t}\right)\right) h(t) \mathrm{d} t \\
& \left.=\int_{0}^{+\infty} E\left(\varphi_{2}\left(A_{T}, B_{T}\right)\right) \mid T=t\right) h(t) \mathrm{d} t \\
& \left.=E\left[E\left(\varphi_{2}\left(A_{T}, B_{T}\right)\right) \mid T\right)\right] \\
& =E \varphi_{2}\left(A_{T}, B_{T}\right)
\end{aligned}
$$

By Lemma 2.3, $A_{T} \geq_{h r} B_{T}$, that is

$$
\left(\max \left\{X_{1}, X_{2}, \cdots, X_{n}\right\}\right)_{(T)} \geq_{h r}\left(\max \left\{Y_{1}, Y_{2}, \cdots, Y_{n}\right\}\right)_{(T)}
$$

Theorem 2.12 Assume that $X$ and $Y$ are independent (not necessarily identical), $T$ is a non-negative random variable, $\mathrm{N}$ be a positive integer-valued random variable which is independent of the $X$ and $Y$, and T, $X$ and $Y$ are independent. If $X \leqslant_{r h} Y$, then

$$
\left(\max \left\{X_{1}, X_{2}, \cdots, X_{N}\right\}\right)_{(T)} \leq_{h r}\left(\max \left\{Y_{1}, Y_{2}, \cdots, Y_{N}\right\}\right)_{(T)}
$$

\section{ACKNOWLEDGMENTS}

This work is supported by the Research Starting Funds for Imported Talents of Kashigar University (No. GCC15ZK-007).

\section{REFERENCES}

[1] Li, X. and Zhang, S.. Comparison between a system of used components and a used system. Journal of Lanzhou University, 2002, 3, 1-8. 
[2] Li, X. and Lu, J. Y.. Stochastic comparisons on residual life and

[3] inactivity time of series and parallel systems. Probability in the Engineering and Informational Sciences,2003, 17, 267-275.

[4] Pellerey,F., Petakos,K. On closure property of the NBUC class under formation of parallel systems. IEEE Trans. Reliab. 2002, 51,452-454.

[5] Shaked, M. and Shanthikumar, J, G. Stochastic Orders and Their Applications. Academic Press, San Diago,1994.

[6] Ross, S. M. Stochastic process, 2nd ed. New York: Wiley, 1996.

[7] Chandra, N. K. and Roy, D. Some results on the reversed hazard rate. Probability in the Engineering and Informational Sciences, 2001,15, 95102.

[8] Block, H., Savits, T. and Singh, H. The reversed hazard rate function. Probability in the Engineering and Informational Sciences, 1998, 12, 6970.

[9] Barlow RE, Proschan F (1981). Statisical Theory of Reliability and LifeTesting. Madison: Silver spring, 1981.

[10] Block, H., Savits, T.,Singh, H. The reversed hazard rate function. Prob. Eng. Inf. Sci.1998,12, 69-70.

[11] Brown, M. Further monotonicity properties for specialized renewal processes. Ann. Probab.1981, 9, 891-895.

[12] Chandra, N.K.; Roy, D. Some results on reversed hazard rate. Prob. Eng. Inf. Sci. 2001, 15, 95-102.

[13] Kayid, M., Ahmad, I.A. On the mean inactivity time ordering with reliability applications. Prob.Eng. Inf. Sci. 2004, 18, 395-409.

[14] Keilson, J., Sumita, U. Uniform stochastic ordering and related inequalities. Can. J. Stat.1982, 10, 181-198.

[15] Li, X., Zuo, M.J. Stochastic comparisons of residual life and inactivity time at a random time. Stochastic Models. 2004, 20 (2), 229-235.

[16] Dewan, I., Khaledi, B.-E., 2014. On stochastic comparisons of residual life time at random time. Statist. Probab. Lett. 88, 73-79. . 